4

6

\section{Correspondent footnote}

18 Michael Bruns, Heinrich-Pette-Institut für Experimentelle Virologie und Immunologie

19 an der Universität Hamburg, Martinistrasse 52, D-20251 Hamburg, Germany. Phone:

20 49-40-48051 231, Fax: 49-40-48051 117, E-mail: mbruns@hpi.uni-hamburg.de. 


\section{Abstract}

25 Although in vitro models of human hepatitis B virus replication are established, so far 26 none could approximate infection efficiency as expected from in vivo observations.

27 Susceptibility for HBV infections has only been reported for primary hepatocytes of

28 human, chimpanzee or Tupaia belangeri and the cell line HepaRG. Here we show that the

29 insusceptible human hepatoma cell line HepG2 can be infected, when the virus was

30 beforehand activated by passage over whole duck liver cell cultures. That suggests an

31 activation step to be performed by specialized liver cells.

32

33

34

35

36

37

38

39

40

41

42

43

44

45

46 
47 The hepatitis B virus (HBV) is a small single stranded DNA-containing enveloped virus.

48 Depending on its relatively strict organotropicity HBV infects mainly hepatocytes and

49 causes severe liver diseases in humans ${ }^{1}$. For this reason it was so far only possible to

50 infect in vitro cultures of primary human or Tupaia belangeri hepatocytes ${ }^{2,3}$, whereas

51 human hepatoma cell lines are usually refractory to HBV infection. We succeeded in

52 infecting the hepatoma cell line HepG2 with HBV by 'activating' the virus in a culture of

53 primary duck liver cells (PDLCs).

54 Using duck HBV (DHBV)-infected PDLCs as a model data were published indicating

55 that eventually scavenging liver sinusoidal endothelial cells (LSECs) play the key role in

56 the initial uptake and targeting of hepadnaviruses in the liver ${ }^{4}$. These data implicated that

57 in the process of transcytosis through LSECs the viral surface of HBV may be altered as

58 a prerequisite for the infection procedure, a step that may be limiting for the infection of

59 hepatoma cell lines like HepG2 (Fig. 1b). Further in vitro experiments performed in our

60 laboratory using preparations of embryonal PDLCs ${ }^{5,6}$ infected with purified DHBV

61 virions ${ }^{7}$ revealed that at least co-staining of single LSECs could be measured using

62 fluorescence-labeled acetylated low density lipoprotein for the identification of LSECs

63 and anti-DHBc antibodies for the localization of the virions (not shown). Taking into

64 account a possibly high conservation of LSEC function we predicted that the human

65 HBV could also be found in association with LSECs in a duck liver cell culture. Indeed,

66 immunofluorescence studies performed with rabbit antisera against $\mathrm{HBC}$ or the human

67 nuclear factor of activated T cells (NFATc1; Santa Cruz) as a marker of LSECs revealed

$6824 \mathrm{~h}$ after the addition of HBV, similarly as described for DHBV-infected PDLCs ${ }^{8}$, co-

69 localization of HBV and NFATc1 in a PDLC culture (Fig. 1a). Cells, negative for 
70 NFATc1 but strongly reacting with anti-HBc antiserum, are possibly Kupffer cells with

71 their typical phagocytic activity. As a source of HBV virions, HepG2.2.15 cells, HepG2

72 cells stably transfected with cloned HBV-DNA, were utilized for the production of

73 infectious $\mathrm{HBV}^{9}$, which was concentrated 50-fold from the collected supernatants ${ }^{10}$.

74 The calculation of virions was performed by DNA dot blot, using a gel-purified full75 length EcoRI fragment of cloned HBV DNA labeled with $\left[\alpha-{ }^{32} \mathrm{P}\right] \mathrm{dCTP}$ as a probe ${ }^{6}$.

76 When we measured the amount of virions after the passage through a PDLC culture, 77 designated as $\mathrm{HBV}^{*}$, against the initial amount of $\mathrm{HBV}$, we found a reduction of about $7860 \%$, supposedly lost by unspecific adsorption and phagocytosis (Fig. 1c). In order to 79 test the infectivity of HBV* for HepG2 cells after passage through primary duck LSECs, 80 a time course kinetic for the release of virions after infection was performed (Fig. 2a). It

81 revealed a steady increase of the amount of viral DNA (again measured by dot blot) in 82 the supernatants of HepG2 cells, comparable to the cell growth-dependent increase of 83 viral output in the stably transfected hepatoma cell line HepG2.2.15. Otherwise, no viral 84 DNA could be detected in the supernatants when untreated HBV was used directly for 85 infection. To study viral DNA replication in infected HepG2 cells in more details we 86 performed a Southern blot using the same radioactively labeled probe as described above.

87 Besides the full-length relaxed circular DNA single-stranded replicative intermediates of

88 HBV DNA were also detected (Fig. 2b). In this study we could find out three further 89 details: first, an incubation time of HBV with LSECs of $24 \mathrm{~h}$ instead of $4 \mathrm{~h}$ did not lead to 90 an increase of infectivity; second, transfer of HBV-incubated activator cells instead of 91 activated virus was also able to initiate HBV-infection; third, to employ another cell type, 92 primary duck kidney cells ${ }^{8}$, as activator cells would not support subsequent infection of 
93 hepatoma cell lines. In a next experiment we replaced every 24 h the culture medium of

94 HBV infected HepG2 cells for the calculation of the amount of the viral surface proteins

95 with a commercially available anti-HBs-coated micro particle enzyme immunoassay

96 (Abbott AXSYM system, Germany) as a measure of virus production per day (Fig. 2c).

97 Seven days after infection the cells were disrupted to reveal HBV proteins after

98 electrophoretical separation over a 5 to $20 \%$ polyacrylamide gradient by Western blots

99 with rabbit antisera raised against the surface proteins (HBs) or the core protein (HBc) of

$100 \mathrm{HBV}$ as described ${ }^{6}$. Only in $\mathrm{HBV}^{*}$-infected cultures the synthesis of HBV core and

101 surface proteins could be demonstrated by immunoblotting (Fig. 2d). The alteration HBV

102 virions acquire during passage through PDLC cultures would supposedly concern

103 envelope protein integrity or reversible modification. We therefore compared their

104 appearance in immunoblotting. The general glycosylation pattern of the viral envelope

105 proteins of $\mathrm{HBV}$ and $\mathrm{HBV}^{*}$ was unchanged. Yet, we found the amount of the

106 unglycosylated small surface protein of HBV to be significantly reduced; whereas the

107 glycosylated as well as the unglycosylated middle and large surface proteins did not show

108 particular alterations (Fig. 1d).

109 We conclude that hepatoma cell lines like HepG2 are susceptible to infection with HBV

110 after a yet to define activation step for HBV virions that takes place in whole liver cell

111 cultures and may even play a significant role in physiological HBV infections. The main

112 suspect for HBV activation would be LSECs as they constitute a permeable barrier

113 between the blood stream and the liver parenchyma and thus necessarily come into close

114 contact with trespassing particles ${ }^{11}$. The absence of morphological features comparable

115 to the liver architecture in cell cultures or defined conditions for the maintenance of 
116 specialized cell types may be the major player that hampers in vitro hepadnaviral

117 infections. Another possibility to influence infectivity of HBV virions would only be

118 secretion of relevant molecules by a cell type in the culture. The establishment of pure

119 LSEC cultures or LSEC cell lines and close pursuance of viral particles within the cells

120 will permit to throw light on their participation in the proposed viral activation.

121

122

123

124 This work was supported by the grant $\mathrm{Br}$ 899/4-1 from the Deutsche 125 Forschungsgemeinschaft and the Bundesministerium für Bildung, Wissenschaft, 126 Forschung und Technologie. The Heinrich-Pette-Institut is supported by the Freie und 127 Hansestadt Hamburg and the Bundesministerium für Gesundheit.

128

129

130

131

132

133

134

135

136

137

138 
References

141 1. Ganem, D. in Fields Virology (eds. Fields, B. N., Knipe, D. M. \& Howley, P. M.)

142 2703-2737 (Lippincott-Raven, Philadelphia, 1996).

1432 2. Gripon, P. et al. Hepatitis B virus infection of adult human hepatocytes cultured in

144 the presence of dimethyl sulfoxide. J Virol 62, 4136-43 (1988).

145 3. Walter, E., Keist, R., Niederost, B., Pult, I. \& Blum, H. E. Hepatitis B virus

146 infection of tupaia hepatocytes in vitro and in vivo. Hepatology 24, 1-5 (1996).

147 4. Breiner, K. M., Schaller, H. \& Knolle, P. A. Endothelial cell-mediated uptake of a hepatitis B virus: a new concept of liver targeting of hepatotropic

5. Kock, J. \& Schlicht, H. J. Analysis of the earliest steps of hepadnavirus replication: genome repair after infectious entry into hepatocytes does not depend on viral polymerase activity. J Virol 67, 4867-74 (1993).

6. $\quad$ Bruns, M., Miska, S., Chassot, S. \& Will, H. Enhancement of hepatitis B virus infection by noninfectious subviral particles. J Virol 72, 1462-8 (1998).

7. Franke, C., Matschl, U. \& Bruns, M. Enzymatic treatment of duck hepatitis B virus: Topology of the surface proteins for virions and noninfectious subviral particles. Virology 359, 126-137 (2007).

8. Maenz, C., Chang, S.-F., Iwanski, A. \& Bruns, M. Entry of duck hepatitis B virus into primary duck liver and kidney cells after discovery of a fusogenic region within the large surface protein. J Virol 81, 5014-5023 (2007).

9. Sells, M. A., Chen, M. L. \& Acs, G. Production of hepatitis B virus particles in Hep G2 cells transfected with cloned hepatitis B virus DNA. Proc Natl Acad Sci U S A 84, 1005-9 (1987).

10. Gripon, P. et al. Infection of a human hepatoma cell line by hepatitis B virus. Proc Natl Acad Sci U S A 99, 15655-60 (2002).

11. Wisse, E., De Zanger, R. B., Charels, K., Van Der Smissen, P. \& McCuskey, R. $\mathrm{S}$. The liver sieve: considerations concerning the structure and function of endothelial fenestrae, the sinusoidal wall and the space of Disse. Hepatology 5 , 683-92 (1985). 
183 Fig. 1. Activation of HBV by PDLCs for infection of human hepatoma cells. (a) Co-

184 immunofluorescence staining of PDLCs after incubation with HBV using anti-HBc and 185 anti-NFATc1 antisera. (b) Model to demonstrate the requirement for activation of HBV 186 by PDLCs before the infection of target cells like HepG2. (c) Analysis of HBV DNA 187 before and after the addition to PDLCs. HBV was added to PDLCs and removed again 188 after an incubation time of $4 \mathrm{~h}$. Calculation of the viral DNAs before and after incubation 189 with PDLCs revealed a loss of HBV virions of about $60 \%$; the presented dot blot data 190 shows the mean of 3 measurements. (d) The same amount of virus particles was used to 191 investigate changes of the viral envelope proteins before and after incubation with 192 PDLCs; GP, glycoprotein, and P, protein, indicate the molecular weight in $\mathrm{kD}$ of the 193 HBV large (L), middle (M), and small surface proteins (S).

194 Fig. 2. Study of viral DNA replication and protein synthesis in HepG2 cells. (a) Dot blot 195 kinetic of viral DNA in the supernatants over 7 days following infection of HepG2 cells 196 with $\mathrm{HBV}^{*}$ and HBV or seeding of HepG2.2.15 cells. (b) Infectivity of HBV* for HepG2 197 cells; prior incubation of HBV with PDLCs was performed for 4 or 24 h (lanes 2 and 3). 198 Alternatively HBV-treated PDLCs or primary duck kidney cells were transferred into the 199 HepG2 culture (lanes 5 and 6); lane 1, HepG2.2.15 cells; RC, relaxed circular, and SS, 
200 single-stranded, DNAs of HBV; M, viral marker DNA. (c) Kinetic study of HBs for one

201 week; here, day 1 represents viral input. All data points represent the mean of 3

202 calculations. (d) Analysis of the production of viral surface (left) or core (21.5 kD)

203 proteins (right) by Western blot one week after infection with $\mathrm{HBV}^{*}$ or $\mathrm{HBV}$; mock*-

204 and mock-treated inputs refer to the supernatants of HepG2 cells similarly passaged over

205 PDLCs or not; P, PDLCs after removal of HBV*.

206
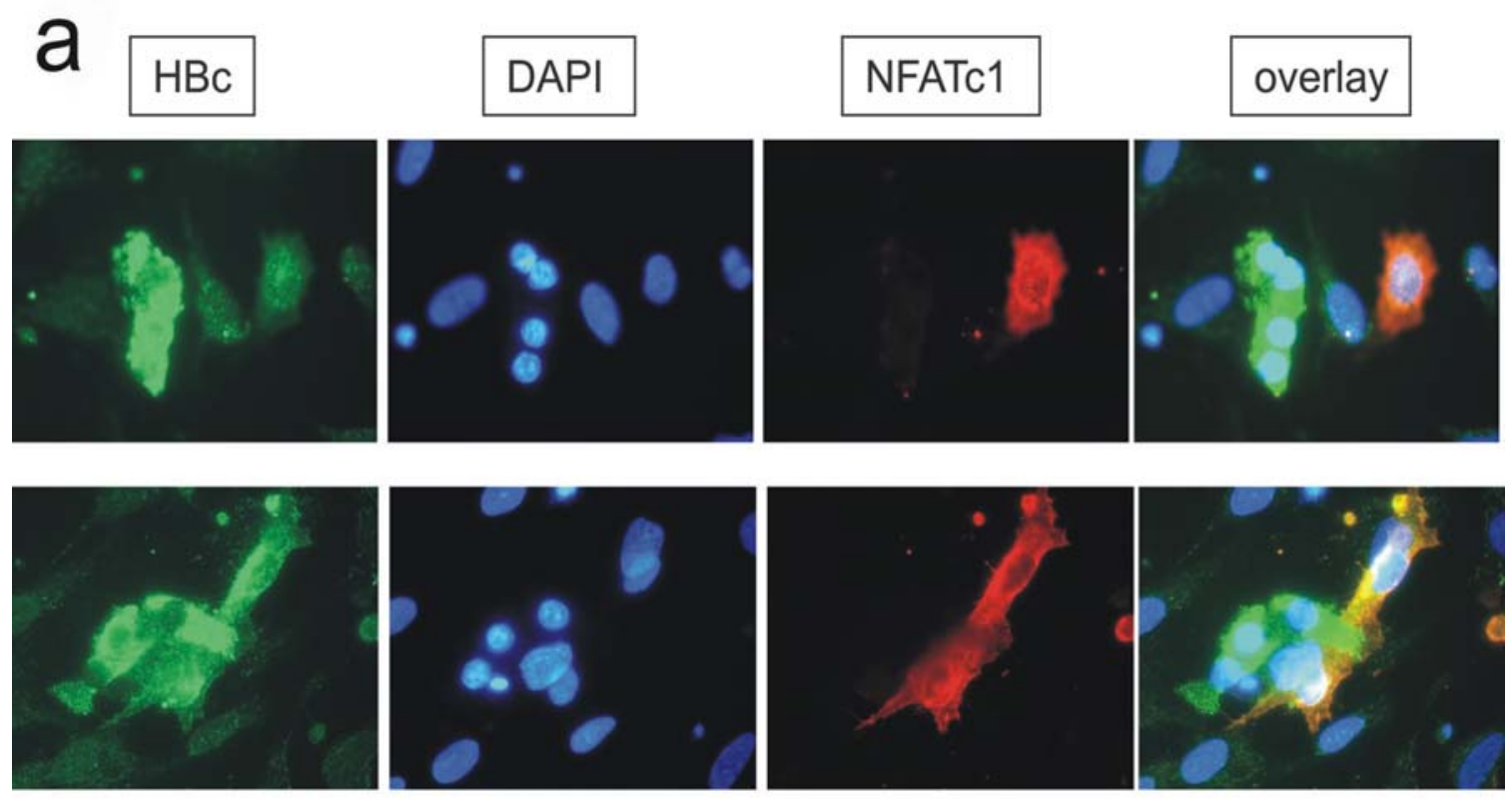

b

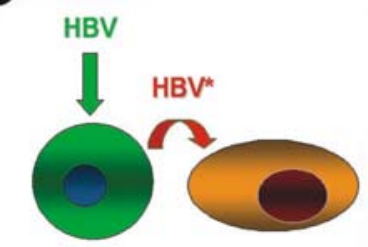

PDLC (LSEC) HepG2 = = activator cell target cell

Fig. 1. Maenz \& Bruns
C

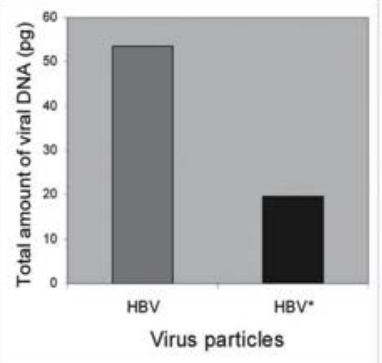

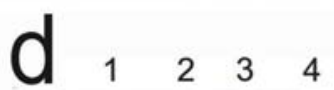

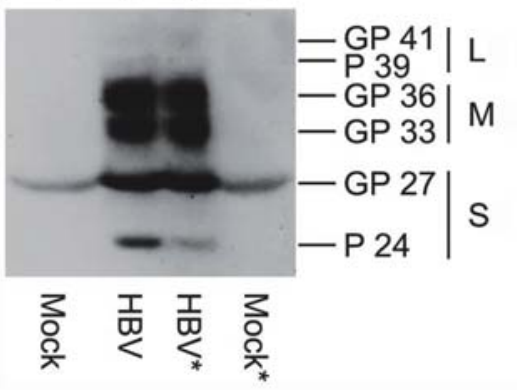



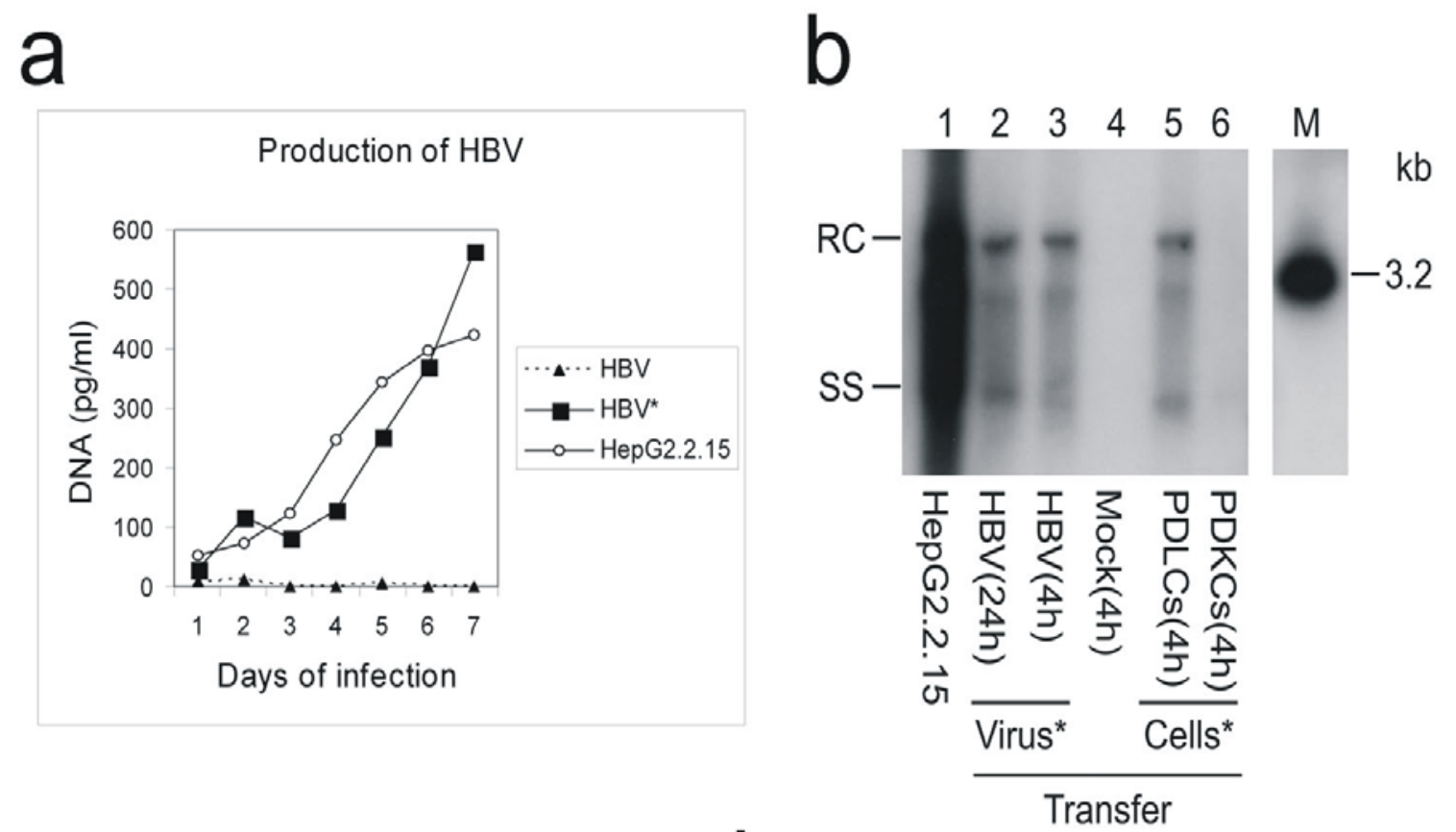

C
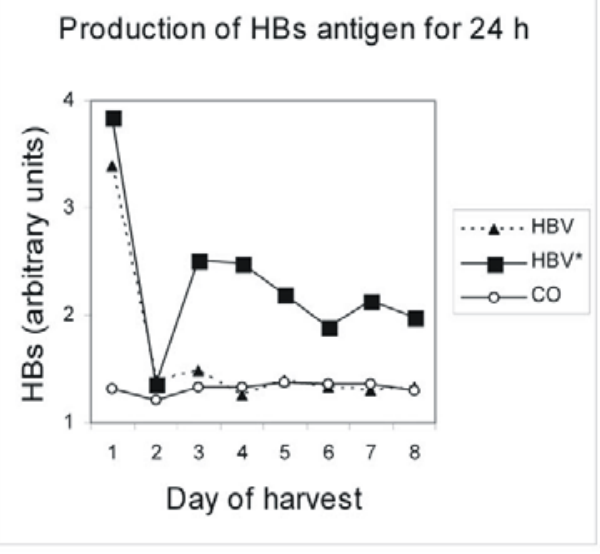

d

Transfer

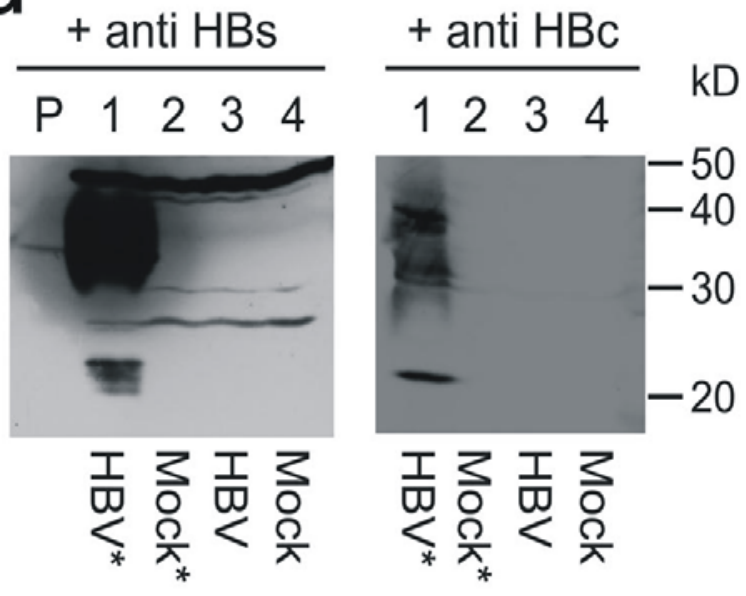

210

211

Fig. 2. Maenz \& Bruns

212 